\title{
Prevalence of varicose veins and chronic venous insufficiency in men and women in the general population: Edinburgh Vein Study
}

\author{
C J Evans, F G R Fowkes, C V Ruckley, A J Lee
}

\begin{abstract}
Study objective-To determine the prevalence of varicose veins and chronic venous insufficiency (CVI) in the general population.

Design-Cross sectional survey. Setting-City of Edinburgh.

Participants-Men and women aged 18-64 years selected randomly from age-sex registers of 12 general practices.

Main results-In 1566 subjects examined, the age adjusted prevalence of trunk varices was $40 \%$ in men and $32 \%$ in women $(p \leqslant 0.01)$. This sex difference was mostly a result of higher prevalence of mild trunk varices in men. More than $80 \%$ of all subjects had mild hyphenweb and reticular varices. The age adjusted prevalence of CVI was $9 \%$ in men and $7 \%$ in women $(p \leqslant 0.05)$. The prevalence of all categories of varices and of CVI increased with age $(p \leqslant 0.001)$. No relation was found with social class.

Conclusions-Approximately one third of men and women aged 18-64 years had trunk varices. In contrast with the findings in most previous studies, mainly conducted in the 1960s and 1970s, chronic venous insufficiency and mild varicose veins were more common in men than women. No evidence of bias in the study was found to account for this sex difference. Changes in lifestyle or other factors might be contributing to an alteration in the epidemiology of venous disease. (F Epidemiol Community Health 1999;53:149-153)
\end{abstract}

Wolfson Unit for Prevention of Peripheral Vascular Diseases, Department of Public Health Sciences, University of Edinburgh, Teviot Place, Edinburgh EH8 9AG

C J Evans

F G R Fowkes

A J Lee

Department of Surgery, Royal Infirmary of Edinburgh C V Ruckley

Correspondence to: Professor F G R Fowkes.

Accepted for publication 15 September 1998
Venous disorders of the legs occur frequently, and range in severity from minor asymptomatic incompetence of venous valves to chronic leg ulceration. Varicose veins are a common manifestation of venous incompetence in the lower limb, and appear as dilated, elongated or tortuous superficial veins. Incompetence of the deep, superficial and/or perforating veins leads to raised venous pressure in the lower leg, which can result in skin changes such as hyperpigmentation and induration with eventual ulceration. These changes in the skin and subcutaneous tissues of the lower leg are often referred to clinically as chronic venous insufficiency (CVI).

Venous disease of the legs causes considerable morbidity and is also costly, with approximately $2 \%$ of national healthcare resources being spent on treatment. ${ }^{1}$ Exactly how common varicose veins are in the community is dif- ficult to determine, because relatively few population-based epidemiological studies have been carried out. Many previous studies have investigated samples of clinic patients or specific occupational groups, and comparison of results is further hampered by a lack of uniform definitions and methods of measurement. ${ }^{2}$ Many of the studies were conducted over 20 years ago and the reported prevalence of varicose veins ranges widely from $0.1 \%$ in women living in rural New Guinea ${ }^{3}$ to $68 \%$ in female chemical workers in Basle, Switzerland. ${ }^{4}$ It is estimated that chronic leg ulceration affects $1 \%$ of the population at some point in their lives 5 and surveys have shown that $57-80 \%$ of leg ulcer patients have demonstrable venous disease. ${ }^{6}$

The Edinburgh Vein Study is the first large scale study of its kind in the United Kingdom to investigate venous disease in the general population. The aims of the study were to determine the prevalence of venous disease of the legs in a randomly selected population sample, and to investigate associations with certain genetic and lifestyle factors. Lifestyles of men and women have changed greatly in the UK since the 1960s and 1970s when many of the previous epidemiological studies were conducted, and such changes might have affected the occurrence of disease. Given recent concerns about the priority accorded to the treatment of varicose veins in the NHS, the purpose of this paper is to provide up to date information on the prevalence of varicose veins and chronic venous insufficiency in the general population.

\section{Methods}

The Edinburgh Vein Study is a cross sectional survey, with a target population comprising men and women aged 18 to 64 years, resident in Edinburgh. An age stratified random sample was selected from the computerised age-sex registers of 12 general practices, whose catchment areas were geographically and socioeconomically distributed throughout the city. A total sample size of 1500 participants was estimated, based on the number required to give an adequate precision for prevalence, to detect a significant difference in prevalence between groups and to enable a subsequent follow up study to be conducted. Details of the methods and response in the Edinburgh Vein Study have been reported. $^{7}$

Subjects attended a clinic in the University of Edinburgh and were examined by one or more members of a research team, comprising 
a nurse, technician, and clinical research fellow. A self administered questionnaire was completed that included personal and occupational details, relevant medical and family history and possible risk factors for venous disease. The method of examination and classification of venous disease used in the Edinburgh Vein Study was adapted from the method used in the Basle Study, ${ }^{8}$ as this was considered to be the best available ${ }^{9}$ and to provide the most detailed classification for the different degrees of varicose veins. During inspection of the legs, subjects stood on a raised platform with their feet in three standard positions: facing towards the examiner with heels together and forefeet spread wide apart, facing away from the examiner in a similar position, and facing away from the examiner with feet parallel. They were asked to remain in a standing position for a minimum of two minutes before examination of their veins, to allow the blood to pool in the legs. Any scars and notable findings on the legs were recorded.

Varices were divided into three types; trunk varices, reticular varices and hyphenweb varices. Trunks were defined as "dilated, tortuous trunks of the saphena magna or parva vein and their branches of the first or second order", reticulars as "dilated, tortuous subcutaneous veins not belonging to the main trunk or its major branches", and hyphenwebs as "intradermal varices". Each of the three groups was sub-divided into grades of severity $1-3$, determined according to the "degree and extent of tortuosity and prominence of the veins". ${ }^{8}$ These grades were determined with reference to photographs from the Basle study. In practice, grade 1 trunks ranged from a small discrete visible or palpable length of dilated trunk vein to more obvious but not grossly dilated varicose veins; grade 2 trunks were more extensive and/or more grossly dilated trunk varices and grade 3 trunks were varices at the most severe end of the spectrum. Each subject was also examined for presence of any pitting ankle oedema, and assessed for CVI as defined in the Basle Study. ${ }^{8}$ Grade 1 CVI was defined as "dilated subcutaneous veins/corona phlebectatica", grade 2 CVI as "hyper- or depigmented areas, with or without corona phlebectatica", and grade 3 CVI as "open or healed ulcus cruris". The recently developed CEAP classification for chronic venous disease ${ }^{10}$ was not available when the Edinburgh Vein Study began. However, the above grades of CVI correspond to the CEAP clinical classification as follows: grade $1 \mathrm{CVI}$ corresponds to Class 1 (malleolar flare), grade 2 CVI corresponds to Class 4 (skin changes), and grade 3 CVI corresponds to Classes 5 and 6 (healed or active ulceration).

As in the Basle Study, ${ }^{8}$ photographs of the legs were taken. The photographic slides were subsequently independently analysed according to the classification system described above, by the two study team members who had not seen the patient in the clinic. Discrepancies of two or more grades between the two observers were resolved by discussion with an experienced clinician (CVR). This process resulted in two independent classifications of the venous status of each subject:- one based on examination in the clinic, and the other on analysis of the photographic slides. The results in this paper are based on the examination findings. In addition, a comparison of the two methods of classification is reported. Quality control measures for this classification procedure included weekly analysis and discussion of the photographic slides, periodic review of reference photographs as a reminder of the original standard and, periodically throughout the study, independent examination of the same volunteers by all three team members to allow identification and discussion of discrepancies.

After each subject's appointment, a report of the clinical findings was sent to their general practitioner. Those subjects wishing more information on varicose veins were offered an advice leaflet ${ }^{11}$ and referred back to their general practitioner. Nineteen subjects had a "home visit" appointment because of their inability to attend the university clinic for medical or social reasons. Local ethics committee approval was given for the study and informed consent was obtained from each study participant.

A survey was carried out of the "nonresponders" (that is, those subjects who initially agreed to participate in the study and subsequently withdrew or failed to attend their appointment, and those subjects who declined to participate in the study from the beginning) from 4 of the 12 practices. This survey took the form of a one page questionnaire, inquiring about past history of venous disease and treatment for varicose veins. For each nonresponder, demographic data were available and a Carstairs deprivation score was derived from the individual's postcode..$^{12} 13$

Information from the recording forms and questionnaires were entered onto a DBASE IV database. The data files were transferred to the Edinburgh University Mainframe computer for analysis using the SPSS-X and SAS statistical packages. The following statistical tests were used:- $\chi^{2}$ test and Mantel-Haenszel test for linear association for categorical data, the MannWhitney test for continuous non-parametric data and the $\kappa$ statistic as a measure of agreement between categorical variables. ${ }^{14}$ The age adjusted prevalence rates were calculated using GLIMMIX (a SAS macro), which fits generalised linear mixed models.

\section{Results}

A total of 1566 subjects attended for examination, 867 women and 699 men, resulting in a response rate of $53.8 \%$ for those contacted and still living in the area. ${ }^{7}$ The age adjusted prevalence of trunk varices (grades 1-3) was 39.7\% in men and $32.2 \%$ in women $(p \leqslant 0.01)$. Table 1 shows that the majority of affected subjects had mild (grade 1) trunks and the difference between the sexes reached statistical significance for grade 1 only $(p \leqslant 0.01)$. Hyphenweb and reticular varices were very common, each affecting over $80 \%$ of subjects, although the majority had these varices only to a mild degree (grade 1). Among the smaller numbers affected 
Table 1 Age adjusted prevalences of grades of varices and chronic venous insufficiency (CVI) by sex

\begin{tabular}{|c|c|c|c|c|c|c|}
\hline & \multirow[b]{2}{*}{ Grade } & \multicolumn{2}{|c|}{$\operatorname{Men}(n=699)$} & \multicolumn{2}{|c|}{ Women $(n=867)$} & \multirow[b]{2}{*}{$p$ value } \\
\hline & & $\%$ & (n) & $\%$ & (n) & \\
\hline \multirow[t]{3}{*}{ Trunk varices } & 1 & 33.3 & $(238)$ & 26.2 & $(223)$ & 0.009 \\
\hline & 2 & 5.4 & (39) & 5.6 & (47) & 0.888 \\
\hline & 3 & 1.0 & (7) & 0.5 & (4) & 0.241 \\
\hline \multirow[t]{3}{*}{ Hypheweb varices } & 1 & 79.2 & (554) & 84.4 & (732) & 0.260 \\
\hline & 2 & 5.9 & $(44)$ & 9.2 & $(76)$ & 0.030 \\
\hline & 3 & 0 & (0) & 0.6 & (5) & - \\
\hline \multirow[t]{3}{*}{ Reticular varices } & 1 & 81.6 & $(571)$ & 85.3 & (739) & 0.422 \\
\hline & 2 & 4.0 & (29) & 6.4 & (54) & 0.042 \\
\hline & 3 & 0 & (0) & 0 & (0) & - \\
\hline \multirow[t]{3}{*}{ CVI } & 1 & 6.9 & (51) & 5.3 & (44) & 0.157 \\
\hline & 2 & 1.3 & (10) & 1.1 & (9) & 0.607 \\
\hline & 3 & 1.0 & (8) & 0.2 & (2) & 0.058 \\
\hline
\end{tabular}

(n) = number in each group with varices/CVI.

by grade 2 hyphenweb and reticular varices, women had significantly higher prevalences than men (both $\mathrm{p} \leqslant 0.05$ ). The age adjusted prevalence of chronic venous insufficiency (grades 1-3) was $9.4 \%$ in men and $6.6 \%$ in women $(p \leqslant 0.05)$. However, sex differences were not statistically significant when the grades of severity were analysed individually $(p>0.05)$. A total of 10 subjects ( 8 men and 2 women) had current or previous venous ulceration (grade $3 \mathrm{CVI}$ ).

Table 2 shows the prevalence of trunk varices by age group. The prevalence increased linearly with age in both sexes, and ranged from $11.5 \%$ in the $18-24$ year olds to $55.7 \%$ in the $55-64$ year olds when both sexes were combined $(p \leqslant 0.001)$. Likewise, the prevalence of both reticular and hyphenweb varices increased linearly with age in both sexes $(p \leqslant 0.001)$. Table 3 shows that, under the age of 35 years, CVI was extremely rare in women and did not occur at all in men. Overall, the prevalence of CVI increased markedly with age $(p \leqslant 0.001)$. In the oldest age group of 55-64 years, the prevalence of CVI was much higher in men $(25.2 \%)$ than women $(12.3 \%)$ ( $p \leqslant 0.001)$. However, even in this age group, most of those affected had only mild (grade 1) CVI (18.3\% of men and 8.6\% of women). The age adjusted prevalence of pitting ankle oedema was $7.4 \%$ in men and $16.0 \%$ in women $(p \leqslant 0.001)$ and increased linearly with age $(p \leqslant 0.001)$.

There was no obvious relation between social class (classified by occupation) and the age and sex adjusted prevalences of either trunk varices or CVI (table 4). The age and sex adjusted prevalence of both conditions was higher in manual workers (social classes IIIM-V) than non-manual workers (social classes I-IIIN) but these differences were not statistically significant (both $\mathrm{p}>0.05$ ).

A slide classification of venous status based on analysis of photographic slides was available for 1555 of the 1566 subjects in the study. The classifications of venous status based on (a) examination in the clinic and (b) analysis of the slides were compared, in each case taking the leg with the higher grade as the patient's score. There was "fair to good agreement" ${ }^{14}$ for trunk varices $(\kappa=0.60)$ and CVI $(\kappa=0.64)$ between the two classification methods.

The age adjusted prevalences of trunk varices and CVI reported by the two principal observers were compared. Observer 1 classified $35.9 \%$ of subjects as having trunk varices and $8.2 \%$ as having CVI, compared with $36.7 \%$ and $8.0 \%$ respectively for observer 2 (both $\mathrm{p}>0.05)$. When further analysed by sex, the age adjusted prevalences of trunks and CVI, as classified by observers 1 and 2 respectively, were as follows; trunks: men $43.2 \% v 42.2 \%$ ( $\mathrm{p}>0.05)$, women $31.2 \%$ v 33.1\% ( $>0.05)$; CVI: men $11.5 \%$ v $10.1 \%$ ( $>>0.05)$, women $6.0 \%$ v $6.8 \%(\mathrm{p}>0.05)$.

There was a total of 1346 "nonresponders"- that is, 998 who refused to participate and 348 who agreed take part in the study but subsequently withdrew. The nonresponders were generally younger, lived in relatively less affluent postcode sectors ${ }^{12}{ }^{13}$ and contained a higher proportion of men than the study attenders (all $\mathrm{p} \leqslant 0.001){ }^{7} \mathrm{~A}$ one page questionnaire inquiring about venous disease was sent to 378 non-responders from 4 of the 12 participating general practices and 194 $(51.3 \%)$ of these questionnaires were returned. There were no significant differences between those who returned and did not return their questionnaires with regard to sex and age band (both $\mathrm{p}>0.05$ ), but the non-responders who returned their questionnaires lived in relatively more affluent postcode sectors than those who did not return them $(p \leqslant 0.05)$. The nonresponders' questionnaire results were compared with the results of study attenders from the same four practices who answered the same

Table 2 Prevalence of trunk varices by age and sex

\begin{tabular}{|c|c|c|c|c|c|c|c|c|c|c|c|}
\hline & \multicolumn{10}{|c|}{$\operatorname{Age}(y)$} & \multirow[b]{2}{*}{$p$ value } \\
\hline & $\begin{array}{l}18-24 \\
\%\end{array}$ & (n) & $\begin{array}{l}25-34 \\
\%\end{array}$ & (n) & $\begin{array}{l}35-44 \\
\%\end{array}$ & (n) & $\begin{array}{l}45-54 \\
\%\end{array}$ & (n) & $\begin{array}{l}55-64 \\
\%\end{array}$ & (n) & \\
\hline Men & 20.0 & (11) & 15.5 & (16) & 36.1 & (57) & 42.0 & (76) & 61.4 & (124) & 0.000 \\
\hline Women & 5.3 & (4) & 13.9 & (22) & 22.6 & (42) & 41.9 & (95) & 50.5 & (111) & 0.000 \\
\hline Total & 11.5 & (15) & 14.6 & (38) & 28.8 & (99) & 41.9 & (171) & 55.7 & (235) & 0.000 \\
\hline
\end{tabular}

$(\mathrm{n})=$ number in each group with varices. Trunk varices include grades 1-3. p values based on Mantel-Haenszel test for linear association. 
Table 3 Prevalence of chronic venous insufficiency (grades 1-3) by age and sex

\begin{tabular}{|c|c|c|c|c|c|c|}
\hline \multirow[b]{2}{*}{ Age (y) } & \multicolumn{3}{|l|}{ Men } & \multicolumn{3}{|c|}{ Women } \\
\hline & (n) & $\%$ & $95 \% C I$ & $(n)$ & $\%$ & $95 \% C I$ \\
\hline $18-24$ & $(0)$ & - & - & (1) & 1.32 & $0.03,7.11$ \\
\hline $25-34$ & (0) & - & - & (2) & 1.25 & $0.15,4.44$ \\
\hline $35-44$ & (4) & 2.53 & $0.69,6.28$ & (7) & 3.76 & $1.48,7.48$ \\
\hline $45-54$ & (14) & 7.73 & $4.31,12.74$ & (18) & 7.93 & $4.87,12.52$ \\
\hline $55-64$ & (51) & 25.25 & $19.25,31.62$ & (27) & 12.27 & $8.17,17.20$ \\
\hline
\end{tabular}

(n) $=$ number in each group with CVI.

Table 4 Age and sex adjusted prevalence of trunk varices and chronic venous insufficiency (CVI) by social class

\begin{tabular}{llllll}
\hline \multirow{2}{*}{ Social class } & \multicolumn{2}{l}{ Trunk varices } & & \multicolumn{2}{l}{ CVI } \\
\cline { 2 - 3 } \cline { 5 - 5 } & $\%$ & $(n)$ & & $\%$ & $(n)$ \\
\hline I & 35.0 & $(43)$ & & 5.6 & $(6)$ \\
II & 35.8 & $(185)$ & & 7.5 & $(39)$ \\
IIIN & 32.2 & $(113)$ & & 7.0 & $(24)$ \\
IIIM & 43.4 & $(91)$ & & 13.3 & $(29)$ \\
IV & 33.4 & $(33)$ & & 10.1 & $(9)$ \\
V & 40.0 & $(32)$ & & 8.1 & $(8)$ \\
\hline
\end{tabular}

Both trunk varices and CVI include all grades (1-3). (n) = number in each group with varices/CVI. Social class based on occupation. Based on a total $=1376$. Excluded are housewives (123), students (56), armed forces (6), unemployed with no previous job (2), and missing data (3).

questions in the main study questionnaire. After adjusting for age and sex, $6.5 \%$ of the 194 non-responders who returned their questionnaire reported a doctor's diagnosis of varicose veins compared with $13.3 \%$ of the attenders $(p \leqslant 0.05)$. Of the men, $6.7 \%$ of nonresponders and $9.1 \%$ of attenders reported a doctor's diagnosis of varicose veins $(p>0.05)$ compared with $6.5 \%$ of non-responders and $16.7 \%$ of attenders among the women $(p \leqslant 0.05)$. Extrapolation of this information to all the non-responders in practices 1-4 suggests that a higher proportion of women than men with a doctor's diagnosis of varices attended, while a similar proportion of men and women without a doctor's diagnosis attended (data not shown).

\section{Discussion}

Results from the Edinburgh Vein Study confirm that venous disease is common in the general population, with approximately one third of the study population showing some degree of trunk varicose veins. In addition, the vast majority of subjects had a mild degree of hyphenweb and reticular varices, suggesting that these minor stigmata are "the norm". An increase in varices with age was demonstrated, in common with findings from many other studies. $^{29}$

The major unexpected finding from this study was the significantly higher prevalence of trunk varicose veins in men compared with women. This significant difference was largely because of a higher prevalence of mild (grade 1) varices in men. Men also had a higher prevalence of chronic venous insufficiency (grades 1-3) than women, although the sex differences were not significant when grades of CVI were analysed individually. Conversely, women had a higher prevalence of moderate (grade 2) hyphenweb and reticular varices, and of pitting ankle oedema, than men.
KEY POINTS

- Trunk varicose veins are common, occurring in approximately one third of the adult population.

- Hyphenweb and reticular varices are "the norm" occurring in over $80 \%$ of the population.

- Mild trunk varices are more common in men than women in this study population $(33.3 \%$ versus $26.2 \%)$ in contrast with findings from many previous studies.

- Prevalence of venous disease is not significantly related to social class.

- Changes in lifestyle may be affecting the epidemiology of venous disease.

Most of the evidence from previous studies indicates a higher prevalence of varicose veins in women. It has been suggested that variation in the sex ratio between different studies may be partly because of differences in the age of the populations studied and the methods of measurement used. ${ }^{9}$ However, many of the results on overall prevalence from previous studies were not adjusted for age, and this factor may have obscured any true sex differences. Also, studies that relied on self assessment of varicose veins or reporting of a previous diagnosis by a physician, may have been prone to bias, because women may have been more likely to report varices or to consult their doctor for this condition than men. This hypothesis is supported by the Edinburgh Vein Study finding that only $10 \%$ of men reported a previous doctor's diagnosis of varicose veins in their questionnaire compared with $17 \%$ of women. Men were subsequently found to have a significantly higher prevalence of trunk varices than women on examination.

The Basle Study (the origin of the method of classification used for the Edinburgh Vein Study) found a similar prevalence of all varices in men and women, but the sample contained relatively few women. ${ }^{8}$ The small number of published studies that have examined population samples have all found a higher prevalence of varices in women than men ${ }^{15-17}$ Abramson et al surveyed residents of a Jewish neighbourhood of Western Jerusalem and found that the age standardised prevalence for varicose veins (excluding venectasias) was $29.5 \%$ in women and $10.4 \%$ in men. ${ }^{15}$ In the Tecumseh Community Health Study, a longitudinal study of a total USA community, the overall prevalence of "any varicose veins" was $25.9 \%$ in women and $12.9 \%$ in men, and although these figures were not age standardised, the prevalence of varices was higher in each age group in women. ${ }^{16}$ Leipnitz et al examined a sample of 2821 subjects aged 45-65 years, randomly selected from the greater Aachen district and found the prevalence of varicose veins to be $29.0 \%$ in women and $14.5 \%$ in men. ${ }^{17}$ However, in a recent study in South Wales in which 600 randomly selected men and women over the age of 60 years were examined, sex was not a significant risk factor for varicose veins (I Harvey, personal communication). In another 
recent study in Bochum, Germany, children were examined on three occasions during their school education. By the age of 18-20 years, men had a higher prevalence of trunk varices, tributary varices, and incompetent perforators than women, while women had a higher prevalence of reticular varices and telangiectasias (hyphenwebs). ${ }^{18}$ Although no tests of significance were reported on these results, the sex differences followed a similar pattern to that seen in the Edinburgh Vein Study.

In the Edinburgh Vein Study, the question that arises therefore is whether the prevalence of varicose veins in men was really higher than in women, or whether this result was caused by bias. Differential measurement error was unlikely because the method used to identify venous disease was based on a standardised classification, ${ }^{8}$ and showed "fair to good" agreement with a second objective classification based on colour photographs. ${ }^{14}$ Furthermore, the two principal observers classified almost identical proportions of men and women as having trunk varices and CVI. Also, in another paper, we have reported that venous incompetence measured by duplex scanning was significantly more common in the deep veins in men than in women. ${ }^{19}$ Alternatively, the results may have been biased if men with varices were more likely to attend than women with varices. However, comparison of the prevalence of doctor's diagnosis of varices in the male and female non-responders and attenders suggests that this was unlikely.

If the prevalence of varicose veins was truly higher in men than women in this population, it may be that changes in lifestyle, working practices or the environment have led to a change in the difference in prevalence between the sexes. The evidence to support any association between varices and many lifestyle factors such as prolonged standing, tight undergarments, toilet posture, chair sitting, and dietary fibre intake is lacking. ${ }^{9}$ However, the available evidence suggests that the prevalence of varicose veins increases with increasing numbers of pregnancies. ${ }^{9}$ Perhaps the general tendency for women to have fewer children later in life has resulted in a lower prevalence of varices in this relatively young group of women. Detailed analysis of the association between lifestyle factors such as pregnancy, hormone use, and dietary fibre intake and the presence of varicose veins and CVI in the Edinburgh Vein Study population will be examined in future papers.

In conclusion, venous disease is a common condition in the young and middle aged. This study suggests that mild varicose veins are more common in men, but there are insuffi- cient data at present to comment on the sex differences in severe venous disease. We have identified no evidence to suggest that the higher prevalence in men is the result of bias in the study, although such bias cannot be ruled out in studies such as this. Follow up of this cohort will provide information on the natural history and development of venous disease in both sexes, and the resulting implications for patients and the provision of healthcare.

Information was kindly provided by Professor L K Widmer and Dr M-T Widmer. The authors would like to thank the following:- Miss M Carson and Mrs E Kerracher for data collection; Mrs L Haggarty for secretarial and administrative support; Mr G Didcock and Mr T Blake for computing support; the general practitioners, practice managers, support staff and patients of the following Edinburgh general practices for their collaboration and participation in the study: Dr White and partners, 21 Chester Street; Milton Surgery; Mackenzie Medical Centre; Ladywell Medical Centre; Bruntsfield Medical Practice; The Long House Surgery; Rose Garden Medical Centre; Crewe Medical Centre; Whinpark Medical Centre; Muirhouse Medical Group and Dr Reid and partners, 25 Mayfield Road.

Funding: The Wellcome Trust.

Conflicts of interest: none.

1 Laing W. Chronic venous diseases of the leg. London: Office of Health Economics, 1992:1-44.

2 Evans CJ, Fowkes FGR, Hajivassiliou CA, et al. Epidemiology of varicose veins - a review. Int Angiol 1994;13:263-70.

3 Stanhope JM. Varicose veins in a population of New Guinea. Int f Epidemiol 1975;4:221-5.

4 Da Silva A, Widmer LK, Martin H, et al. Varicose veins and chronic venous insufficiency - prevalence and risk factors in 4376 subjects of the Basle Study II. Vasa 1974;3:118-25.

Callam MJ, Harper DR, Dale JJ, et al. Chronic ulcer of the eg: clinical history. BMf 1987;294:1389-91.

6 Alexander House Group. Consensus paper on venous leg ulcers. Phlebology 1992;7:48-58.

7 Evans CJ, Fowkes FGR, Ruckley CV, et al. Edinburgh Vein Study: Methods and response in a survey of venous disease in the general population. Phlebology 1997;12:127-35.

8 Widmer LK, ed. Peripheral venous disorders - prevalence and socio-medical importance. Bern:Hans Huber, 1978

9 Callam MJ. Epidemiology of varicose veins. Br F Surg 1994; 81:167-73.

10 Porter JM, Moneta GL and an International Consensus Committee on Chronic Venous Disease. Reporting standards in venous disease: an update. $\mathcal{F}$ Vasc Surg 1995;21:63545

11 Health Education Board for Scotland. Help and advice on leg problems - varicose veins. Coping with long term conditions. Edinburgh.

12 Carstairs V, Morris R. Deprivation and health in Scotland. Aberdeen: Aberdeen University Press, 1991.

13 McLoone P. Carstairs scores for Scottish postcode sectors from the 1991 Census. Glasgow: Public Health Research Unit, the 1991 Census. Glasgow: P

14 Fleiss JL. Statistical methods for rates and proportions. 2nd ed. New York: Wiley, 1981.

15 Abramson JH, Hopp C, Epstein LM. The epidemiology of varicose veins - A survey of western Jerusalem. $\mathcal{F}$ Epidemiol Community Health 1981;35:213-17.

16 Coon WW, Willis PW, Keller JB. Venous thromboembolism and other venous disease in the Tecumseh community health study. Circulation 1973;48:839-46.

17 Leipnitz G, Kiesewetter P, Waldhausen P, et al. Prevalence of venous disease in the population: first results from a prospective study carried out in greater Aachen. In: Davy A, Stemmer R, eds. Phlebology '89. Paris: John Libby Eurotext, 1989:169-71.

18 Schultz-Ehrenburg U, Weindorf N, Matthes U, et al. New epidemiological findings with regard to initial stages of epidemiological findings with regard to initial stages of
varicose veins (Bochum study I-III). In: Raymondvaricose veins (Bochum study I-III). In: RaymondParis: John Libbey Eurotext, 1992:234-6.

19 Evans CJ, Allan PL, Lee AJ, et al. Prevalance of venous reflux in the general population on duplex scanning: The Edinburgh Vein Study. $\mathcal{F}$ Vasc Surg (in press). 